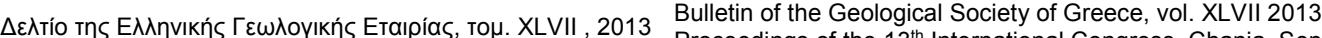
Proceedings of the $13^{\text {th }}$ International Congress, Chania, Sept.

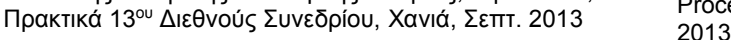

\title{
ANALYTICAL GEOCHEMISTRY IN THE SERVICE OF MEDICINE: AN EXPERIMENTAL STUDY OF URINARY STONES FROM NORTHERN GREECE
}

\author{
Iordanidis A. ${ }^{1}$ and Garcia-Guinea $\mathbf{J .}^{2}$ \\ ${ }^{I}$ Department of Geotechnology and Environmental Engineering, Technological Educational \\ Institute (TEI) of Western Macedonia, Kila, 50100 Kozani, Greece, aiordanidis@yahoo.co.uk and \\ andior@teikoz.gr \\ ${ }^{2}$ Museo Nacional Ciencias Naturales, CSIC, Abascal 2, 28006 Madrid, Spain
}

\begin{abstract}
Knowledge of the precise human biomineral composition may allow physicians to recommend an appropriate prophylactic therapy for the patient and thus prevent or delay the stone recurrence. The present study focuses on the application of complementary analytical techniques to the characterization of human urinary stones. Several gallbladder and renal stone samples were obtained from patients dwelling in areas of northern Greece. A comprehensive analytical study took place, employing the following, common in analytical geochemistry, techniques: Environmental Scanning Electron Microscopy (ESEM) coupled to Energy Dispersive System (EDS), X-Ray Diffraction (XRD), thermogravimetry (TG), $\mu$ Raman spectroscopy and Cathodoluminescence (CL). A detailed determination of morphological, micro-structural, molecular, chemical and mineralogical characteristics of the urinary stone samples was achieved. It was evident by our study the application of powerful analytical techniques could substantially help the medical advisors to ascribe a medical treatment of diseases related to stone formation.
\end{abstract}

Keywords: Human Calculi, ESEM-EDS, Raman, XRD, Cathodoluminescence.

\section{Пері́ $\eta \psi \eta$}

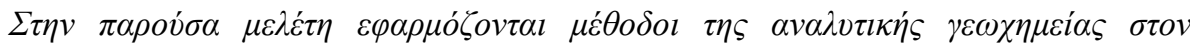

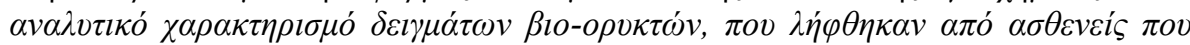

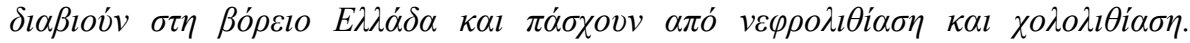

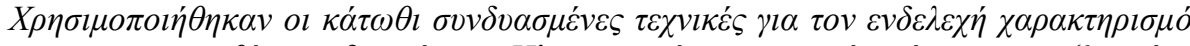

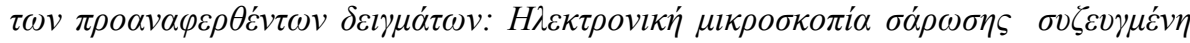

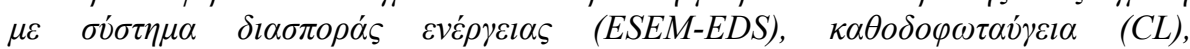

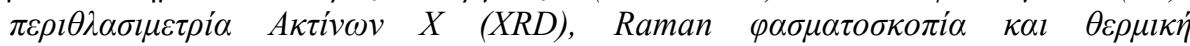

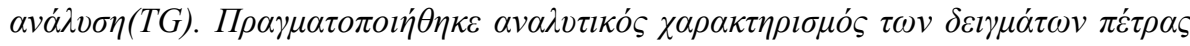

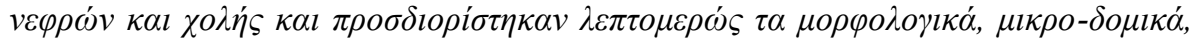

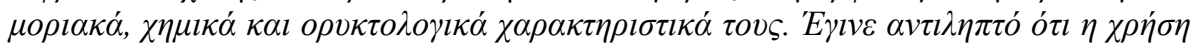

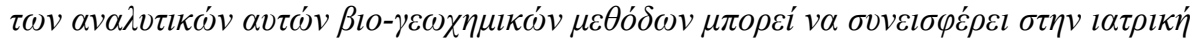

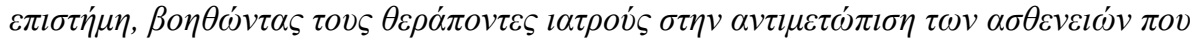

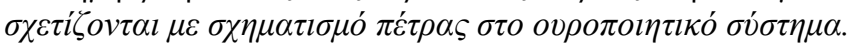

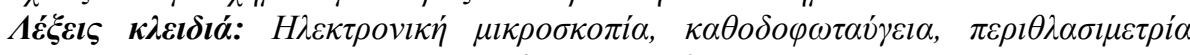

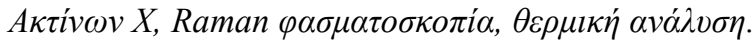

$\underline{\text { XLVII, No } 2-818}$ 


\section{Introduction}

Urinary stones are generally comprised of organic and inorganic compounds (mainly of calcium, phosphate, magnesium salts, oxalate, and uric acid). They occur in varieties of shape, size and crystalline to amorphous structure and range in color from creamy white, yellow, black and brown. There is also variation in the size of stones ranging from few millimeters to $5 \mathrm{~cm}$. The pathogenesis of human stones is complex and is not completely understood.

An elevated number of nephrolithiasis and cholelithiasis (i.e. formation of renal and gallbladder stones respectively) incidents has been observed among patients dwelling in northern Greece, especially within Western Macedonia province (Iordanidis et al., 2010, 2011). This increasing frequency, related to different epidemiological factors and diseases, prompted us to carry out an analytical investigation on this kind of human stones. Such analytical studies, incorporating both non-destructive and destructive analytical methodologies, have been conducted worldwide, providing significant information on the formation and composition of human calculi and helping the physicians to take prophylactic measures for the patients (Grunhage and Lammert, 2006; Abboud 2008a, 2008b).

In the present study, the application of complementary analytical techniques, such as Environmental Scanning Electron Microscope coupled to Energy Dispersive System (ESEMEDS), $\mu$ Raman spectroscopy, X-Ray Diffraction (XRD), Thermogravimetry (TG) and Cathodoluminescence (CL) is attempted in order to provide a more comprehensive determination of the renal and gallbladder stones.

\section{Materials and Methods}

Several urinary (renal and gallbladder) samples were supplied by the surgeons of the General Public (Mamatsio) Hospital of Kozani and the Central (Genimatas) Hospital of Thessaloniki, northern Greece. The stones were washed and dried before analysis. Samples were cut and two types of block sections were prepared: polished ones, which were used for the Scanning Electron Microscopy and $\mu$ Raman spectroscopy and unpolished ones for the X-Ray Diffraction (XRD) and Cathodoluminescence (CL) analyses. Moreover, a tiny amount of each sample was ground for the thermal analyses.

A Philips QUANTA 200 Environmental Scanning Electron Microscope (ESEM), coupled with an Oxford INCA Energy 200 Energy Dispersive System (EDS) was used for the chemical analyses and the recording of pictures, under back-scattered electron (BSE) mode.

Cathodoluminescence (CL) spectra were collected on polished slabs, at low vacuum mode without coating to keep open way out to the CL emission, using a Gatan MonoCL3 detector with a PA-3 photomultiplier attached to the ESEM.

The Raman spectra of samples were recorded with a Thermo Scientific DXR Raman Microscope with a $780 \mathrm{~nm}$ laser beam, which has a point-and-shoot Raman capability of 1- $\mu \mathrm{m}$ spatial resolution, setting the power value of the sample irradiation at $12 \mathrm{~mW}$. The average spectral resolution in the Raman shift range of $100-3000 \mathrm{~cm}^{-1}$ was $5 \mathrm{~cm}^{-1}$.

For the X-ray diffraction (XRD), a Phillips PW1710/00 diffractometer was used with a CuKa radiation source, equipped with a graphite monochromator. The XRD patterns were obtained by step scanning from $2^{\circ}$ to $64^{\circ}$ ( $2 \theta$ in steps of $0.020^{\circ} ; 4 \mathrm{~s}$ per step) and compared with the XRD card files of the Joint Committee on Powder Diffraction Standards.

Thermogravimetry (TG) analyses were recorded with a thermal analyzer Model 851e Mettler Toledo in $\mathrm{N}_{2}$ atmosphere. Thermal treatments were performed at a heating rate of $10^{\circ} \mathrm{C} \mathrm{min}{ }^{-1}$ from room temperature up to $1000^{\circ} \mathrm{C}$. 


\section{Results and Discussion}

The macroscopic views of four characteristic gallbladder and one renal calculi, which were analyzed in our study, are shown in Figure 1.

The first gallstone is a 'black pigment' urinary stone with a spherulitic, fragile and porous structure. The ESEM images (Figure2) reveal a massive appearance with micro nodules. The chemical composition of this gallstone, as revealed by EDS analysis, consists of $\mathrm{CaO}(44.17 \%)$ and $\mathrm{CO}_{2}(52.15 \%)$, indicating the presence of calcium carbonate minerals. The Raman spectra of two different regions of observation revealed two types of minerals, a calcium carbonate (most favourably ascribed to aragonite) and a bilirubin phase. Finally, the XRD pattern (Figure 4-1) shows a characteristic hump between $10^{\circ}$ and $30^{\circ}$ of $2 \theta$ angle, ascribed to amorphous organic phases and aragonite mineral, with its characteristic peaks of d-spacing $(3.35,3.27,1.97$ and 1.87 $\AA)$.
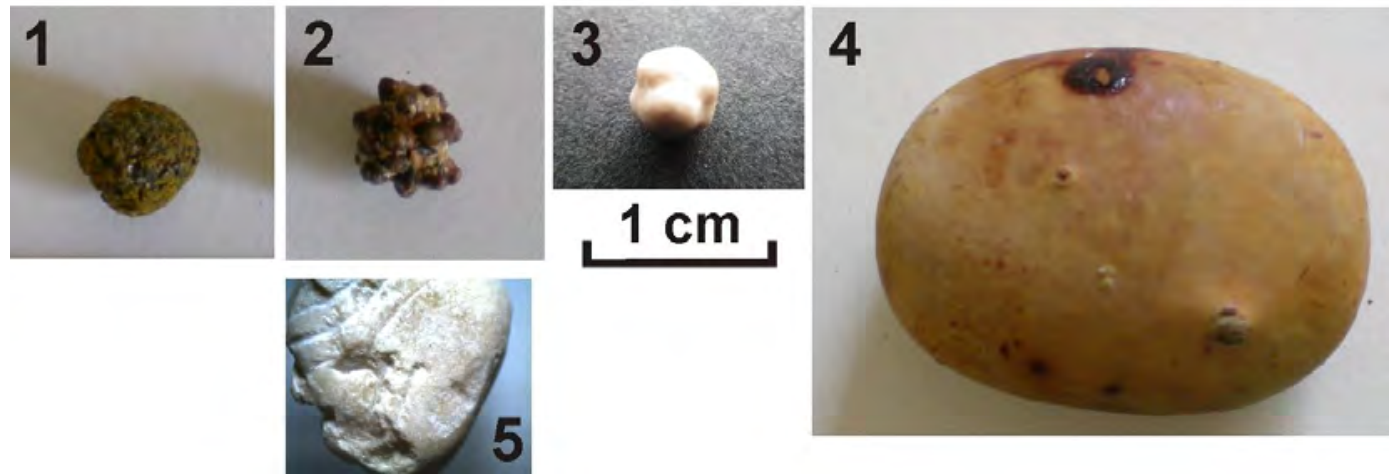

Figure 1 - Macroscopic images of the four gallbladder (No 1 - 4) and one renal (No 5) stones analyzed in this study.

The second sample in an asteroidal assemblage of pale-coloured nodules. The concentric structure is more evident under ESEM observation (Figure 2). Based on the chemical composition (EDS) of distinct layers within the gallbladder, we can conclude that the inter-bedded layers have a variable composition of calcium carbonate, calcium hydroxyl-apatites and Ca-oxalates. The phosphorousrich areas are in fact grains intercalated in the concentric matrix. A typical Ca-oxalate mineral (either whewellite $\left[\mathrm{CaC}_{2} \mathrm{O}_{4} \cdot \mathrm{H}_{2} \mathrm{O}\right.$ )] or weddellite $\left[\mathrm{CaC}_{2} \mathrm{O}_{4} \cdot 2 \mathrm{H}_{2} \mathrm{O}\right]$ ) was determined with Raman spectroscopy. The XRD analysis of this sample revealed amorphous organics along with carbonate (characteristic peaks of d-spacing at 5.87, 3.64 and $2.97 \AA$ ) and oxalate (peak at $5.19 \AA$ ) minerals.

The third sample is a white spherical calculus, with a size of approximately $5 \mathrm{~mm}$ and rather dense and hard comparing to the previous ones. The ESEM/EDS analysis inferred a purely organic $\left(\mathrm{CO}_{2}\right.$ $100 \%$ material (Figure 2). The Raman spectroscopy revealed a very distinct molecule of cholesterol with sharp and clear peaks (Figure 3). The XRD pattern of the same sample provided information of a mixture of cholesterol crystalline structures (characteristic peaks of d-spacing at $5.21,4.11$ and $4.89 \AA$ ) and organic-rich phases, most probably assigned to oxalic acids (peak at $5.56 \AA$ ). Precipitation of cholesterol from supersaturated bile leads to the gallstone formation and is related to several different pathways, which in turn include nucleation, crystallization, growth and agglomeration of cholesterol crystals (Jayalakshmi et al., 2009; Athanasiadou et al., 2013).

The fourth gallstone is approximately $3 \mathrm{~cm}$ long with a pale colour and a pebble-like appearance. An ideal concentric structure is shown in the ESEM image (Figure 2). Numerous layers are interbedded. The growth of the gallbladder calculus occurs around an initially formed nucleus. The EDS analysis determined a gallstone rich in carbon $\left(\mathrm{CO}_{2} 68.34 \%\right)$ and nitrogen $\left(\mathrm{N}_{2} \mathrm{O}_{5} 31.44 \%\right)$. The Raman spectra revealed a well-crystallized molecule of uric acid. Uric acid is a heterocyclic compound of carbon, nitrogen, oxygen, and hydrogen with the chemical formula $\mathrm{C}_{5} \mathrm{H}_{4} \mathrm{~N}_{4} \mathrm{O}_{3}$. This 
is also confirmed by the XRD analysis (Figure 4), in which very distinctive peaks of uric acid crystals are revealed (peaks at 6.55, 5.61. 3.85, 3.27, 3.10 and $2.24 \AA$ ). Whereas it is usual to have variation in composition from the core to the outer surface of such mixed calculi (Wilson et al., 2010), in our study, several zones throughout the calculus were analyzed by EDS and Raman spectroscopy, indicating a composition of a rather pure uric acid.
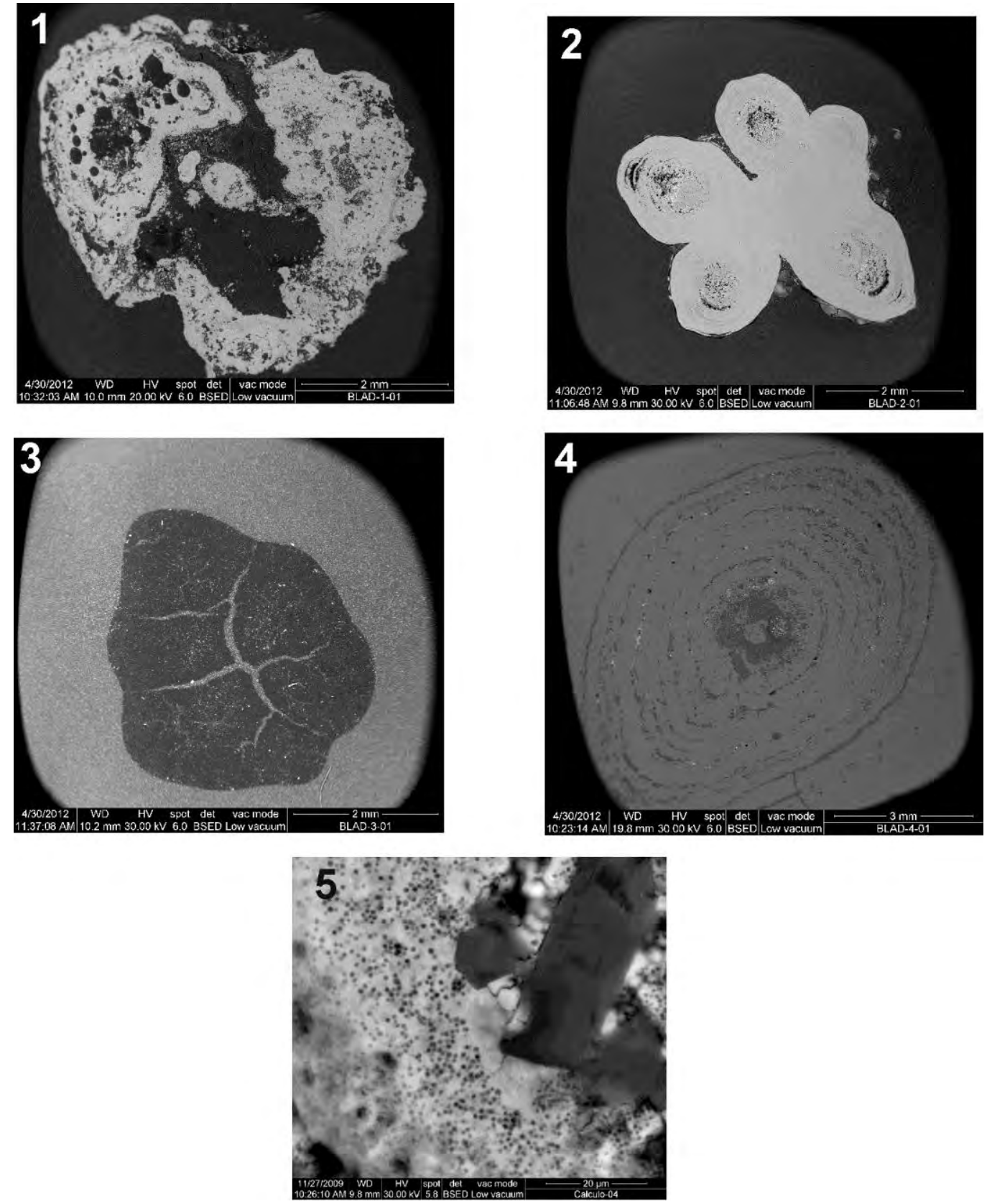

Figure 2 - ESEM images of five urinary stones: (1) calcium-rich gallbladder No1; (2) gallbladder No2, rich in apatites, calcium carbonates and oxalates; (3) the organic-rich gallstone No 3; (4) the amino-acidic gallstone No 4; (5) hexagonal cystine crystals (dark) and microbial remnants (white pores) of the renal stone No5 . 


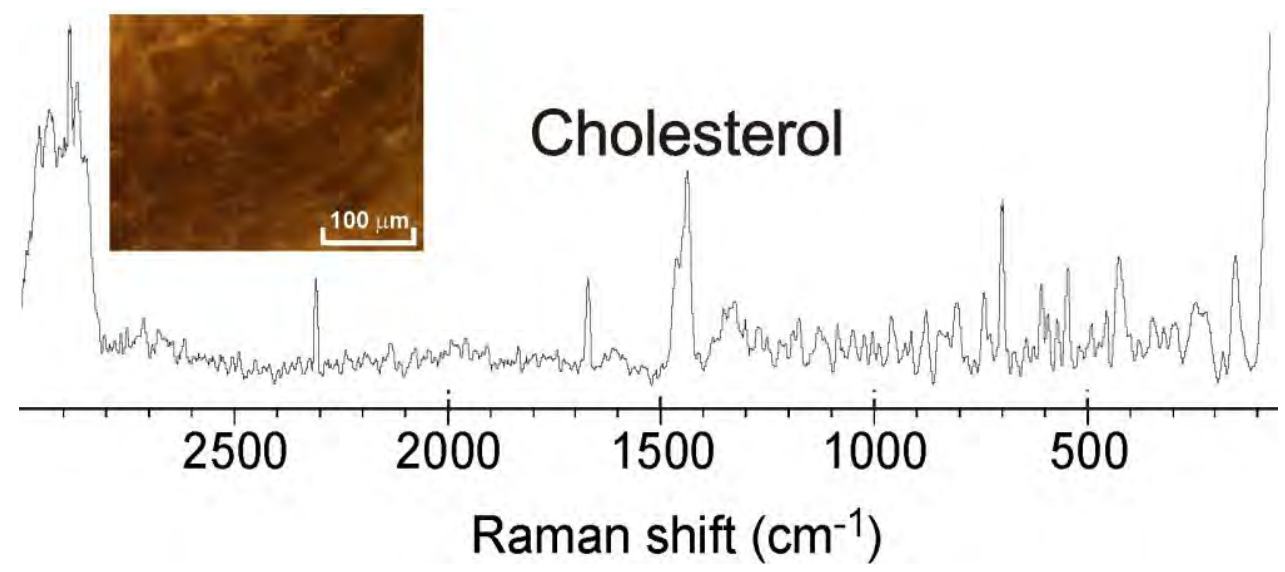

Figure 3 - Raman spectrum and microscopic views using the con-focal Raman microscope of the cholesterol sample No 3 .

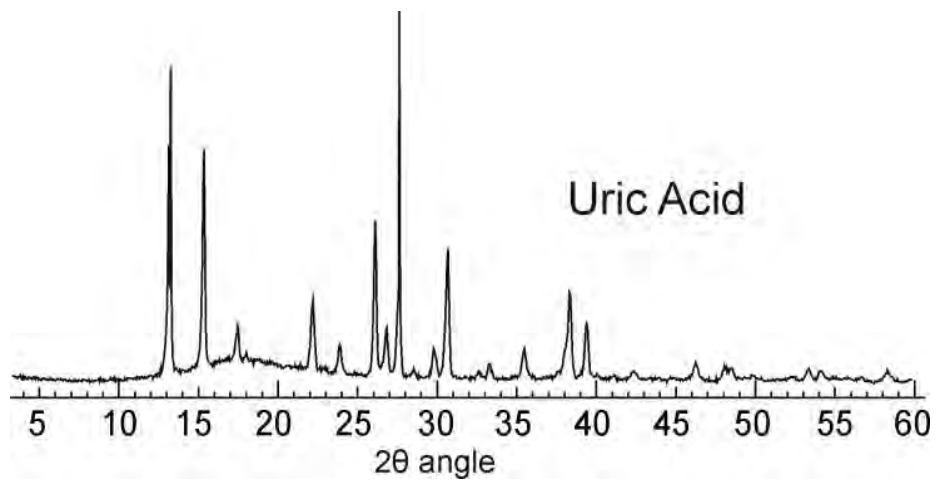

Figure 4 - Characteristic XRD peaks of the Uric Acid crystals of sample No 4.

A characteristic concentric texture is clearly shown for the No5 renal calculus. Using ESEM, thick cystine layers inter-bedded with thin calcium hydroxyl-apatite layers are observed. The elevated concentrations of sulphur are clearly shown under EDS, while calcium and phosphorous prevail within the apatite regions. Characteristic hexagonal cystine crystals are observed under higher magnification (Figure 2). The mineralogical (XRD) analysis revealed a clear L-cystine structure (the less soluble amino-acid found in the urine) (Figure 4). The thermal analysis revealed the characteristic endothermic peak at $246{ }^{\circ} \mathrm{C}$ found in L-cystine and a high amount of mass loss $(90 \%)$, as expected for such an organic compound (Figure 5).

The cathodoluminescence (CL) technique was employed as a complementary method to all the aforementioned techniques (ESEM-EDS, Raman, XRD, TG) to investigate the calculi samples. To our knowledge, there has not been published any CL analytical results on human stones in the global. Thus, it could be regarded as a first attempt to utilize the cathodoluminescence spectroscopy for the analysis of gallbladder calculi. Figure 6 contains characteristic CL spectra of the mineral constituents of the cystine calculus No5. Cathodoluminescence spectra were obtained from several areas of the stone. The chemically pure collagen displays similar and more intense CL plots than hydroxyapatite, thus producing a significant mask of the CL emission, whereas the CL emission peaks at 325 (L-cystine-apatite) and $310 \mathrm{~nm}$ (Ca-oxalate) could be associated to oxygen hole centre emission defects coupled with $\mathrm{C}-\mathrm{OH}$ precursor bonds. For the gallbladder stones (No 1-4), in general, two intensive peaks are determined in all the CL spectra, one between 300 and $310 \mathrm{~nm}$, with an intensity varying between 3800 and 8000 a.u., and another rather broad between 600 and $620 \mathrm{~nm}$, with a considerably lower intensity. These analytical data of 
cathodoluminescence could be regarded as reference for any related future studies, notwithstanding that our $\mathrm{CL}$ experiments are launching cathodoluminescence technique as a significant analytical technique for biomaterials characterization.

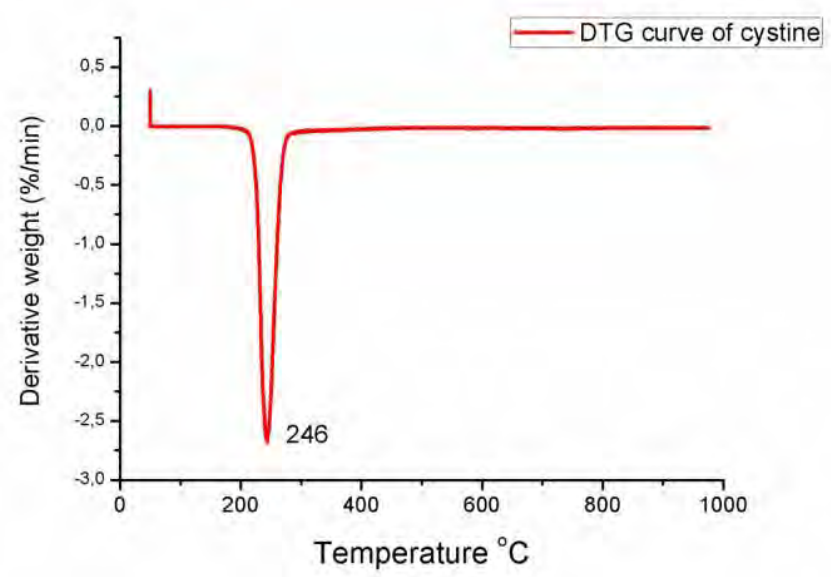

Figure 5 - Derivative Thermogravimetric (DTG) curve of cystine calculus No5.

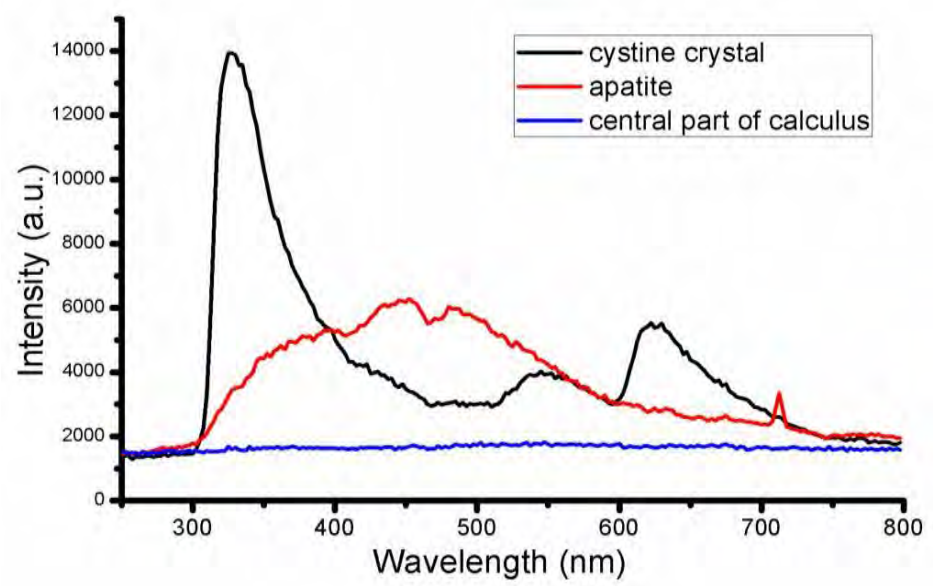

Figure 6 - CL emission spectra of three characteristic areas of cystine calculus No5: central calculi part, apatite mineral and cystine crystal.

In synopsis, the employment of complementary methodologies provided a comprehensive characterization of the human calculi. It is noticeable that certain constituents could not be detected by one single methodology, a fact that clearly demonstrates the significance of using combined techniques such as the electron microscopy imaging and EDS chemical analysis, microRaman molecular spectroscopy, X-ray Diffraction for the detection of crystalline phases and the Cathodoluminescence probe for the relationships between defects and emission. Our study proves that the application of powerful analytical techniques could substantially help the medical advisors. In particular, having a thorough micro-chemical and structural analysis of an urinary stone, the medical treatment of diseases related to stone formation could be better scheduled. Knowledge of the precise bio-mineral composition may allow physicians to recommend an appropriate prophylactic therapy for the patient and thus prevent or delay the cystine stone recurrence. 


\section{Acknowledgements}

The technical staff from the Museo Nacional Ciencias Naturales, CSIC, Madrid (Rafael Gonzalez Martin, Manuel Castillejo, Laura Tormo Cifuentes, Marta Furió Vega, Alberto Jorge García, Cristina Paradela) for their help in conducting the analyses are gratefully acknowledged. We are also indebted to the physicians form the National Hospital of Kozani (Giousef Chisam, Angelopoulos Angelis and Doulgeralis Michael) and Dr. Papadopoulou Lambrini from the School of Geology, Aristotle University of Thessaloniki, for their collaboration.

\section{References}

Abboud I.A. 2008a. Concentration effect of trace metals in Jordanian patients of urinary calculi, Environmental Geochemistry and Health, 30, 11-20.

Abboud I.A. 2008b. Mineralogy and chemistry of urinary stones: patients from North Jordan, Environmental Geochemistry and Health, 30, 445-463.

Athanasiadou D., Godelitsas A., Sokaras D., Karydas A.G., Dotsika E., Potamitis C., Zervou M., Xanthos S., Chatzitheodoridis El., Chye Goo, H. and Becker U., 2013. New insights into the chemical and isotopic composition of human-body biominerals. In: Cholesterol gallstones from England and Greece, Journal of Trace Elements in Medicine and Biology, $27(2), 79-84$.

Grunhage F. and Lammert F. 2006. Pathogenesis of gallstones: a genetic perspective, Best Practice \& Research Clinical Gastroenterology, 20 (6), 997-1015.

Iordanidis A., Garcia-Guinea J., Correcher V. and Goundas I., 2011. Optical and spectral observations on cystine, oxalate and apatite renal calculi, Spectroscopy Letters, 44 (7-8), 490-494.

Iordanidis A., Garcia-Guinea J. and Goundas, I., 2010. Microchemical and microstructural characteristics of cystine (a renal stone), XIX Congress of the Carpathian Balkan Geological Association, Thessaloniki, Greece, 23-26 September 2010, pp. 166

Jayalakshmi K., Sonkar K., Behari A., Kapoor V.K. and Sinha N, 2009. Solid state 13C NMR analysis of human gallstones from cancer and benign gall bladder diseases, Solid State Nuclear Magnetic Resonance, 36, 60-65.

Wilson E.V., Junaid Bushiri M. and Vaidyan V.K. 2010. Characterization and FTIR spectral studies of human urinary stones from Southern India, Spectrochimica Acta Part A, 77, 442445 . 